\title{
PTH-126 CHANGES IN ELF SCORE DURING TREATMENT WITH PEGYLATED INTERFERON AND SILYMARIN ARE ASSOCIATED WITH PROGRESSION OF HISTOLOGICAL LIVER DISEASE IN NON-RESPONDER PATIENTS WITH CHRONIC HEPATITIS C
}

doi:10.1136/gut.2011.239301.527

S Tanwar, 1, ${ }^{*}$ E Ellis, ${ }^{2}$ J Parkes, ${ }^{2}$ C Herold, ${ }^{3}$ W Rosenberg' ${ }^{1}$ Centre for Hepatology, University College London, London, UK; ${ }^{2}$ University of Southampton, Southampton, $U K{ }^{3}{ }^{3}$ University of Erlanghen, Erlangen, Germany

Introduction Liver biopsy remains the reference standard for monitoring changes in liver fibrosis resulting from antifibrotic therapy. While non-invasive serum markers have been validated in the detection of fibrosis in patients with chronic liver disease, their utility in tracking changes in fibrosis is not established.

Methods 70 previous non-responders to pegylated interferon and ribavirin (40 male, 30 female, age range $24-67$, mean 48.7) were recruited from five centres. Patients were randomised to receive pegylated interferon with or without silymarin as an antifibrotic therapy for 24 months.

All patients underwent a liver biopsy and ELF tests (HA, P3NP, TIMP-1) prior to and after treatment (month 0 and 24) and area under receiver operating characteristic curves plotted.

ELF tests were also taken during treatment (month 12). Changes in histological fibrosis stages before and after therapy (0-24 months) were compared with changes in ELF scores before and during therapy ( $0-12$ months).

Results ELF test results correlated well with liver histology with AUROC of 0.832 and 0.830 (F0-1 vs F2-6), 0.847 and 0.802 (F0-2 vs F3-6), 0.811 and 0.858 (F0-3 vs F4-6), 0.868 and 0.891 (F0-4 vs F5-6) pretreatment and post-treatment respectively.

Following treatment, the absolute change in Ishak score ranged from -4 to +4 , mean -0.027 , SD 1.51 with 21 patients with increased and 24 with decreased fibrosis.

At month 12 the changes in ELF score ranged from -1.02 to 2.85, mean 0.5911, SD 0.834 .

The change of ELF between 0 and 12 months was found to correlate with the absolute change of Ishak fibrosis stage on biopsy before and after treatment $(R=0.315, p=0.004)$.

Conclusion ELF testing performed well when compared with liver biopsy prior to and after treatment.

A significant association was observed between change of ELF test at 12 months during therapy and histological change after 24 months of therapy. This suggests that a change in ELF score during therapy may predict changes in histology over a longer period. If confirmed, this may permit the refinement of 'response guided therapy' by identifying those patients who will benefit from both continued and prolonged treatment.

Competing interests None.

Keywords antifibrotic, biomarker, ELF, fibrosis, hepatitis C virus. 